\title{
Passivation of all-angle black surfaces for silicon solar cells.
}

\author{
Tasmiat Rahman $^{\mathrm{a}^{*}}$, Ruy S. Bonilla ${ }^{\mathrm{b}}$, Amirjan Nawabjan ${ }^{\mathrm{a}}$, Peter R. Wilshaw ${ }^{\mathrm{b}}$, Stuart A \\ Boden $^{\mathrm{a}}$
}

${ }^{a}$ Electronics \& Computer Science, University of Southampton, Southampton, SO17 1BJ, United Kingdom

${ }^{\mathrm{b}}$ Department of Materials, University of Oxford, Oxford, OX1 3PH, United Kingdom

\begin{abstract}
Optical losses at the front surface of a silicon solar cell have a significant impact on efficiency, and as such, efforts to reduce reflection are necessary. In this work, a method to fabricate and passivate nanowire-pyramid hybrid structures formed on a silicon surface via wet chemical processing is presented. These high surface area structures can be utilised on the front surface of back contact silicon solar cells to maximise light absorption therein. Hemispherical reflectivity under varying incident angles is measured to study the optical enhancement conferred by these structures. The significant reduction in reflectivity $(<2 \%)$ under low incident angles is maintained at high angles by the hybrid textured surface compared to surfaces textured with nanowires or pyramids alone. Finite Difference Time Domain simulations of these dual micro-nanoscale surfaces under varying angles supports the experimental results. In order to translate the optical benefit of these high surface area structures into improvements in device efficiency, they must also be well passivated. To this end, atomic layer deposition of alumina is used to reduce surface recombination velocities of these ultra-black silicon surfaces to below $30 \mathrm{~cm} / \mathrm{s}$. A decomposition of the passivation components is performed using capacitance-voltage and Kelvin Probe measurements. Finally, device simulations show power conversion efficiencies exceeding $21 \%$ are possible when using these ultra-black Si surfaces for the front surface of back contact silicon solar cells.
\end{abstract}

Keywords- Nanowire, black silicon, surface passivation, silicon solar cells, dielectric thin films, corona discharge.

\section{Introduction}

In order to improve the efficiency of silicon $(\mathrm{Si})$ solar cells, the front surface can be designed with antireflective (AR) structures that enhance carrier generation in the cell. Current approaches to reducing top surface reflectance for monocrystalline silicon cells use thin film coated micron-scale pyramidal structures formed from alkaline solutions that etch preferentially along certain $\mathrm{Si}$ crystal planes. For solar cells made from multicrystalline silicon wafers, where the random nature of the grain orientations precludes the formation of pyramids, acid texturing is instead used Whilst these methods are industry standards, new approaches have emerged to improve on optical absorption by utilizing nanoscale texturing. Examples include nano-textured surfaces formed via laser ablation [1,2], reactive ion etching [3, 4], and wet chemical etching [5-9]. Several solar cells with nanowire AR structures have recently been reported with high power conversion efficiencies. Savin et al. created a nanowire solar cell using deep reactive ion etching (DRIE) with an efficiency of 22.1\% [10]. This approach to texturing has also grown in interest recently due to emergence of diamond-wire sawing which complicates traditional methods for texturing of multicrystalline silicon [11]. Ingenito et al. [12] demonstrated the use of dualtextured black Si (nano-cones on micro-pyramids), fabricated also via plasma etching, on back contact solar cells, achieving power conversion efficiencies up to $19.1 \%$. Recently, dual-textured black Si

\footnotetext{
* Corresponding author:
} 
surfaces created using solution-based processes to form nanowire-pyramid hybrid structures have been shown to exhibit average reflectance $<2 \%$ for light incident perpendicular to the surface [13]. The simplicity of producing this ultra-black Si makes it promising for photovoltaic applications.

The top-down fabrication of silicon nanowires using solution processing has risen in popularity as a cost effective and practical way of producing high aspect ratio nanowires with near-vertical sidewalls, compared to lithography and etching methods [14]. In particular, the metal assisted chemical etch (MACE) process, whereby silicon nanowires are formed using a noble metal and hydrofluoric acid (HF) has attracted much attention for several reasons. Firstly, it is a simple and cost-effective method of etching various Si nanowires with controllable parameters. Almost all of the processes can be done in chemical facilities without the need for expensive and complicated equipment. Secondly, MACE is much more flexible and can be used to make high surface-to-volume ratio structures with various crosssectional shapes in comparison to vapour-liquid-solid (VLS) based methods that can only be used to grow wires with circular cross sections [15]. Si nanowires fabricated by MACE generally possess high crystalline quality, although the surface roughness of these nanowires is higher compared to VLS-grown nanowires. Compared to dry etching (e.g., RIE), chemical-based etching is considered a gentler process which tends to introduce fewer surface defects in the remaining material [16]. Recently, Oh et al. reported an $18.2 \%$ cell efficiency for a MACE textured front junction device [17]. Fang et al. fabricated an ultra-thin solar cell with nanowires created by MACE and achieved a power conversion efficiency of $16.6 \%$ [18]. The challenge for solution-processed black Si lies in achieving adequate passivation to mitigate the increase in surface recombination due to the large increase in surface area.

A common approach used to passivate standard textured n-type surfaces in solar cells is the use of thermally grown $\mathrm{SiO}_{2}$ and plasma enhanced chemical vapour deposition (PECVD) of $\mathrm{SiN}_{\mathbf{X}}[19]$. This proves difficult with nanowire textures due to loss of material in the thermal oxide case, and poor conformity in the PECVD nitride case. Passivation of nano structures, however, is of utmost importance since significant surface recombination arises from the increased surface area and near surface defects present after nanowire texturing. Despite the difficulties in using CVD films, Davidsen et al [20] recently presented a laser-doped selective emitter (LDSE) cell with a full-area, screen-printed aluminium back surface field featuring a PECVD $\operatorname{SiN}_{\mathrm{x}}$ passivated black silicon front surface that demonstrated a power conversion efficiency exceeding $18 \%$. The best performance achieved to date is that reported by Savin et al. [10] where $22.1 \%$ efficient black silicon interdigitated back-contacts cells were achieved. In their work, aluminium oxide formed by atomic layer deposition (ALD) was used to circumvent the surface recombination issue in black silicon. Several recent studies have shown that ALD is the most suitable technique to fully cover and passivate nanostructured surfaces [21, 22].

In this work, dual-scale nanowire-pyramid hybrid structures on silicon wafer surfaces are first characterized optically, with a focus on the optical benefit observed as the angle of incidence is varied. This study highlights the significant benefits of these structures over the use of nanowires or pyramids alone, the AR performance of which has been shown to degrade significantly with increasing angles [23]. An integrating sphere with an automated rotation stage is employed to characterize the fabricated surfaces. Furthermore, finite difference time domain (FDTD) modelling of the structures is used to support the experimental work. Alongside the optical study, we use an $\mathrm{ALD} \mathrm{Al}_{2} \mathrm{O}_{3}$ coating to passivate nanowire-pyramid hybrid surfaces. Using a combination of capacitance-voltage, Kelvin probe, corona discharge, and lifetime measurements, the key components of surface passivation are analysed and recombination velocities below $30 \mathrm{~cm} / \mathrm{s}$ are demonstrated. Finally, device simulations demonstrate that the performance of IBC cells can exceed $>21 \%$ when using these passivated, antireflective surfaces.

\section{Experimental methodology}




\subsection{Textured surface fabrication}

The fabrication of the hybrid surfaces involves alkaline etching to form a random pyramidal surface, followed by a metal assisted chemical etch (MACE) to decorate the pyramids with a dense array of nanowires. The MACE process mechanism is as follows: A silicon substrate partly covered by a noble metal, silver (Ag) in this case, is subjected to an etchant of HF. Silicon beneath the noble metal is etched much faster compared to the surrounding silicon. In this work, MACE was carried out using silver nanoparticles as the metal catalyst, provided in the form of silver nitrate $\left(\mathrm{AgNO}_{3}\right)$ solution. To fabricate the hybrid surface (i.e. pyramids decorated with nanowires), the substrate (6" N-type Si wafer, Cz, 2.5$5 \Omega \mathrm{cm},<100>$ ) is first cleaved into $5 \mathrm{~cm} \times 5 \mathrm{~cm}$ square samples and then cleaned using a piranha etch solution $\left(\mathrm{H}_{2} \mathrm{SO}_{4}+\mathrm{H}_{2} \mathrm{O}_{2}\right)$. The cleaned samples are then textured with random microscale pyramids using a solution of $\mathrm{KOH}(0.2 \mathrm{M})$ and IPA at $70^{\circ} \mathrm{C}$ for $60 \mathrm{~min}$. The nanowires are then fabricated using a single solution of diluted silver nitrate $\left(\mathrm{AgNO}_{3}, 0.06 \mathrm{M}\right)$ and $\mathrm{HF}(3 \mathrm{M})$ in a ratio of $1: 1$. The etch time is 2.5 mins. The solution is held at a temperature of $50^{\circ} \mathrm{C}$. This process is a one-step etch process which uses temperature to regulate the etch rate, in order to have good control over the length of the nanowires. This process is beneficial over the two step process, which uses hydrogen peroxide as a catalyst, as a high etch rate results in porous Si rather than well-defined nanowires when fabricating structures of sub-micron height.

\subsection{Surface passivation coating}

Surfaces textured with pyramids only, nanowires only and the pyramid-nanowire hybrid structures were coated with aluminium oxide $\left(\mathrm{Al}_{2} \mathrm{O}_{3}\right)$ using atomic layer deposition (ALD), Cambridge Nanotech Savannah. ALD is capable of depositing highly uniform and conformal thin films by alternating exposures of a surface to vapours of two chemical precursor reactants [24]. The substrate was held a temperature of $200{ }^{\circ} \mathrm{C}$ and trimethyl aluminium (TMA) and water were used as the reactants. The first reactant, TMA, reacts with the surface layer of the Si substrate forming a saturated monolayer. The volatile products of this reaction (TMA vapour and methane) are subsequently removed and water vapour (the second reactant) is introduced which forms a saturated monolayer of oxygen. The products of this reaction are then pumped away, leaving behind a thin layer of $\mathrm{Al}_{2} \mathrm{O}_{3}$. The cyclic process is repeated until the desired thickness is reached. Conformal coverage can be achieved on high aspect ratio structures (i.e. the nanowires fabricated in this work) due to the films saturating to a monolayer with each precursor pulse. Further advantages of ALD compared to chemical vapour deposition (CVD) or sputtering are its repeatability (low sensitivity to variations of precursor flux [24]) and scalability (many wafers can be tightly stacked and batch processed in one chamber). In this work, 170 cycles were used with a precursor diffusion time of $10 \mathrm{~s}$ per $\AA$ of deposition in order to achieve conformal coverage of the black Si.

The surface passivation quality provided by dielectric films is given by two components. First, the capacity to chemically remove dangling bonds, thus interface trap states; second, the concentration of fixed charged that induces an electric field which repels one sign of carriers from the surface and prevents them recombining. In alumina films, it is well known that a post deposition anneal activates the passivation quality by improving both the field effect and the chemical components of passivation [25]. To test this, alumina coated specimens underwent a post deposition anneal for 30 mins at $400^{\circ} \mathrm{C}$ in a $\mathrm{N}_{2}$ atmosphere $(500 \mathrm{sccm})$. The contribution of the field effect component of passivation, before and after annealing, was evaluated by externally depositing charge using a corona discharge apparatus. This set-up used a point to plane voltage of 20-30 kV and distance of $20 \mathrm{~cm}$. Further description of the corona rig used can be found in [26]. 


\subsection{Characterisation}

Reflectance measurements were undertaken using an integrating sphere technique. This is a spherical cavity with inner walls coated with a near-ideal Lambertian diffuse reflector, resulting in equal light scattering in all directions. This causes light in the sphere to uniformly illuminate the interior wall such that a detector placed at one of the ports on the sphere can sample a representative proportion of the total amount of light. Light is coupled from a port on the sphere into an Ocean Optics USB2000 + VISNIR-ES spectrometer. The light source is a tungsten-halogen HL-2000-FHSA from Ocean Optics. The sample $(2 \mathrm{~cm}$ by $2 \mathrm{~cm})$ is placed at the centre of the sphere by attaching it to a clip suspended from the rotation stage in order to allow the angle of incidence of the incoming light to be changed. A polished silicon sample of the same dimensions as the textured silicon samples was used as a reflectance standard and reflectance was calculated relative to this standard. This measurement set-up is illustrated in Figure 1. Hemispherical reflectance spectrometry at an angle of incidence of 8 degrees was performed using a Bentham PVE300 photovoltaics characterisation system, using a Spectralon reflectance standard.

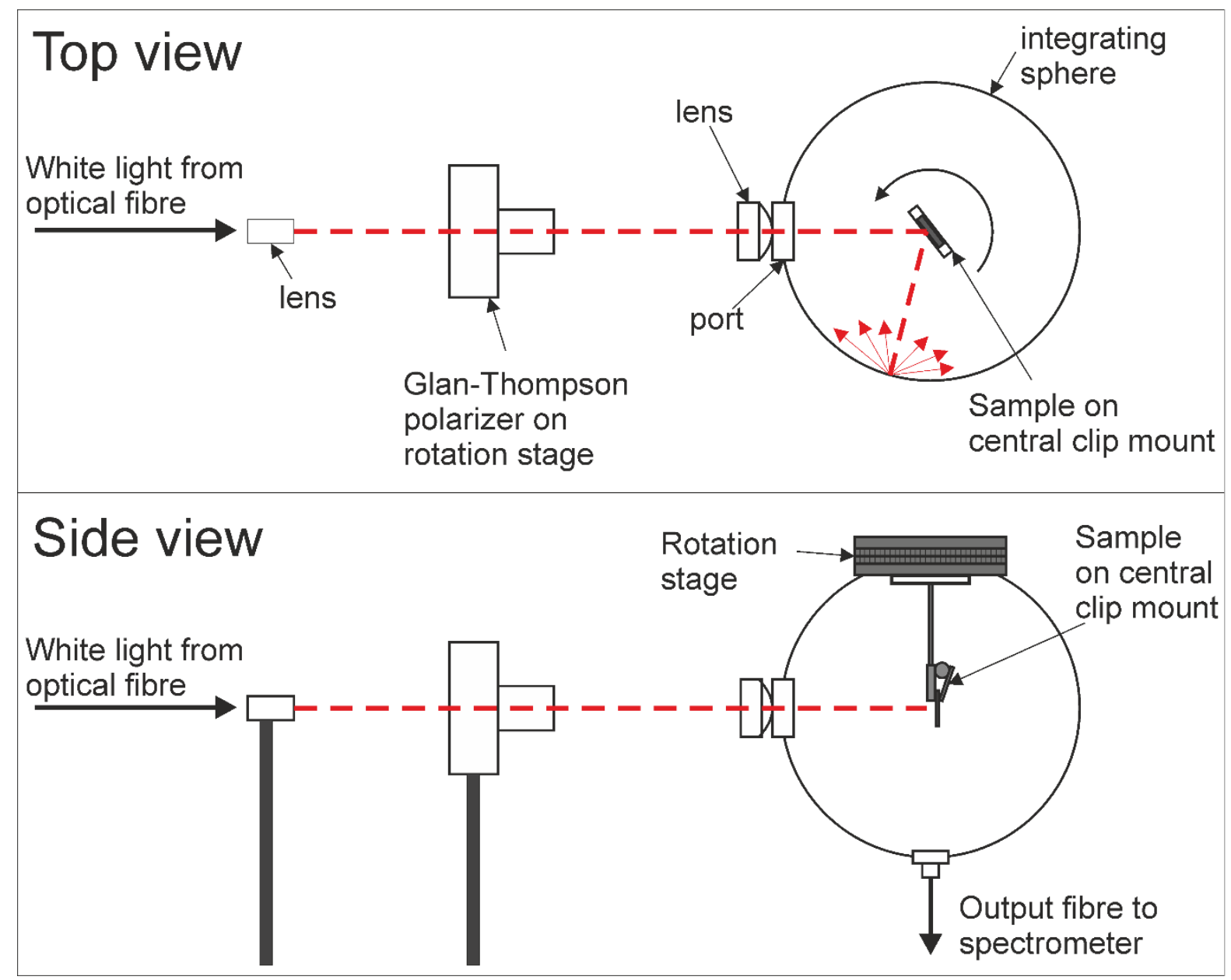

Figure 1: Integrating sphere set-up with in-built rotation stage for angular measurements

Surface morphology was characterised using scanning electron microscopy (NVision40, Carl Zeiss). Surface recombination was assessed by photo-conductance decay lifetime measurements in a Sinton WCT 120 instrument using transient and quasi-steady state modes [27]. Lifetime samples were prepared 
by producing the same surface morphology on the front and back surface of $5 \mathrm{~cm} \times 5 \mathrm{~cm} \mathrm{n}$-type 2.5-5 $\Omega \mathrm{cm} \mathrm{Cz}$ silicon samples. High frequency capacitance-voltage $(\mathrm{CV})$ was performed in an Agilent E4980AL Precision LCR Meter. Kelvin probe (KP) measurements were carried out using a modified Scanning Kelvin Probe from KP Technology as described in [28].

\section{Optical results}

\subsection{Simulation of reflectance and power absorption}

The textured surfaces are modelled to analyse the performance over a range of incident angles. Frequently-used techniques to simulate sub-wavelength optical structures include rigorous coupledwave analysis (RCWA), finite element method (FEM) and finite difference time domain (FDTD). The latter has the benefit of efficient broadband 3D modelling and is thus useful for solar applications which require the optical response from an AM1.5 input spectrum. The commercial Lumerical FDTD Solutions package is used for the simulations performed in this work. Literature on FDTD simulations of NWs has already shown significant reduction in reflection which is attributed to guided modes, Bloch modes and Fabry-Perot resonances [29-31]. Furthermore, it is shown that the geometrical properties of the NW arrays (NWAs) strongly influence the emergence of such modes [13]. Optical modelling of micro-nano surfaces has shown increased absorption due to the superposition of the optical benefits of the pyramid (multiple reflections) and the NWs (guided modes and Fabry-Perot resonances) [13]. In this paper we extend the aforementioned work and analyse the influence under varying angles to illustrate the benefit of the hybrid surface when considered over a wide range of incident angles.

\subsubsection{Simulation set-up}

The FDTD technique solves electromagnetic fields as a function of time and then applies Fourier transforms to calculate the spectral response. Simulations undertaken with perpendicular plane wave injection (incident angle $0^{\circ}$ ), can utilize the broadband plane wave source as well as periodic boundary conditions. However, as this study analyses the influence of plane waves under varying angles of incidence, the boundary conditions in the $x-y$ region are changed to Bloch boundaries and the wavelength is a fixed value per simulation. The wavelength is fixed because the simulated angle of incidence of the source changes as a function of frequency for non-zero injection angles [24]. Bloch boundary conditions are used as these take into account phase change across the unit cell. The plane wave source is injected from the top of the simulation domain. The boundary conditions in the $\mathrm{z}$ direction are perfectly matched layers (PML) to model a semi-infinite substrate. Under steep angles, whereby reflections from the PML layers can increase, a larger number of PML layers is needed to retain accuracy in the simulations [32]. Alongside the wavelength and angle of incidence, the polarization angle is also set $\left(0^{\circ}\right.$ for $\mathrm{P}$ and $90^{\circ}$ for $\left.\mathrm{S}\right)$. The use of fixed wavelengths, Bloch boundary conditions, increased PMLs and taking into account polarisation, significantly increases simulation time and complexity compared to that of perpendicular injection simulations of the hybrid surfaces shown elsewhere [13]. A fitted model of multi-coefficients is used to represent tabulated refractive index $(\mathrm{n}, \mathrm{k})$ data of silicon and the minimum length of the mesh cell is set to $10 \mathrm{~nm}$, respectively. A termination criteria of 1e-3 is used to confirm that the spectral response obtained by the Fourier transformation is valid. A data monitor is placed in the $x-y$ plane at the top of the simulation domain to measure the reflected power, and cross-sectional monitors are placed along the $\mathrm{x}-\mathrm{z}$ and $\mathrm{y}-\mathrm{z}$ planes in the middle of the simulation domain to map the spatial absorption profiles of the simulated structures.

\subsubsection{Simulation results}


The simulated reflectance spectra of a periodic NWA (normalised to flat-Si) under varying angles, $\theta$, is shown in Figure 2. The case is shown for 3 different NW diameters ( $D=50 \mathrm{~nm}, 100 \mathrm{~nm}, 150 \mathrm{~nm})$ of a fixed height $(500 \mathrm{~nm})$, as well 3 different heights $(\mathrm{H}=500 \mathrm{~nm}, 1 \mu \mathrm{m}, 2 \mu \mathrm{m})$ of a fixed diameter (100 $\mathrm{nm})$. The periodicity of these NWs is $200 \mathrm{~nm}$. The figure illustrates the modes supported by the structures in which the NW is analogous to a circular dielectric waveguide [33]. For D $=50 \mathrm{~nm}$, only the fundamental mode is supported and thus reflectivity is low for $\lambda<450 \mathrm{~nm}$. For larger $\lambda$, the NW has less impact and the spectra are similar to that of flat $\mathrm{Si}$. As the diameter increases, higher modes are supported hence reflectivity drops across the spectrum. The presence of Fabry-Perot resonances between the NW/air interface and the NW/bulk-Si interface becomes more prevalent as the NW height increases. The plots show that such Fabry-Perot resonances shift with $\theta$, and that reflectivity for larger $\lambda$ increases more than shorter $\lambda$, with increasing angle of $\theta$.

Spatially mapped power absorption in cross-section through a pseudo-random NWA and hybrid structure is shown in Figure 3 for $\lambda=650 \mathrm{~nm}$ and $\theta=10^{\circ}, 40^{\circ}$ and $70^{\circ}$. The calculated average absolute reflectivity (R) is also given on each plot as a percentage. The pseudo-random NWA is a better representation of the variance in diameter of NWs fabricated using the MACE method. The pseudorandom NWA has a unit cell size of $5 \mu \mathrm{m}$ by $5 \mu \mathrm{m}$ in the $\mathrm{x}-\mathrm{y}$ plane. This contains $625 \mathrm{NWs}$ with diameters ranging from $40 \mathrm{~nm}$ to $180 \mathrm{~nm}$ and a height of $1 \mu \mathrm{m}$. The hybrid case has the same unit cell size but also consists of a pyramid (base width $=5 \mu \mathrm{m}$, height $=3.536 \mu \mathrm{m}$ ), with NWs distributed across the pyramid facets. The results show that hybrid structures outperform (exhibit a lower reflectivity than) the NWs alone. This remains the case across all $\theta$. The superior reduction in reflectivity observed in the hybrid case is due to the combination of the optical benefits of the pyramid (multiple reflections) and the NWA (guided modes and Fabry-Perot resonances). The increase in reflection in the NWA fabricated on a planar surface, at large $\theta \mathrm{s}$, is countered in the hybrid case as the reflections can be directed onto nanowires of an adjacent hybrid structure. This low reflectivity across a range of $\theta$ is beneficial for photovoltaic devices as the incident angle of light on a solar panel is dependent on module orientation and location, and can change considerably over time. 

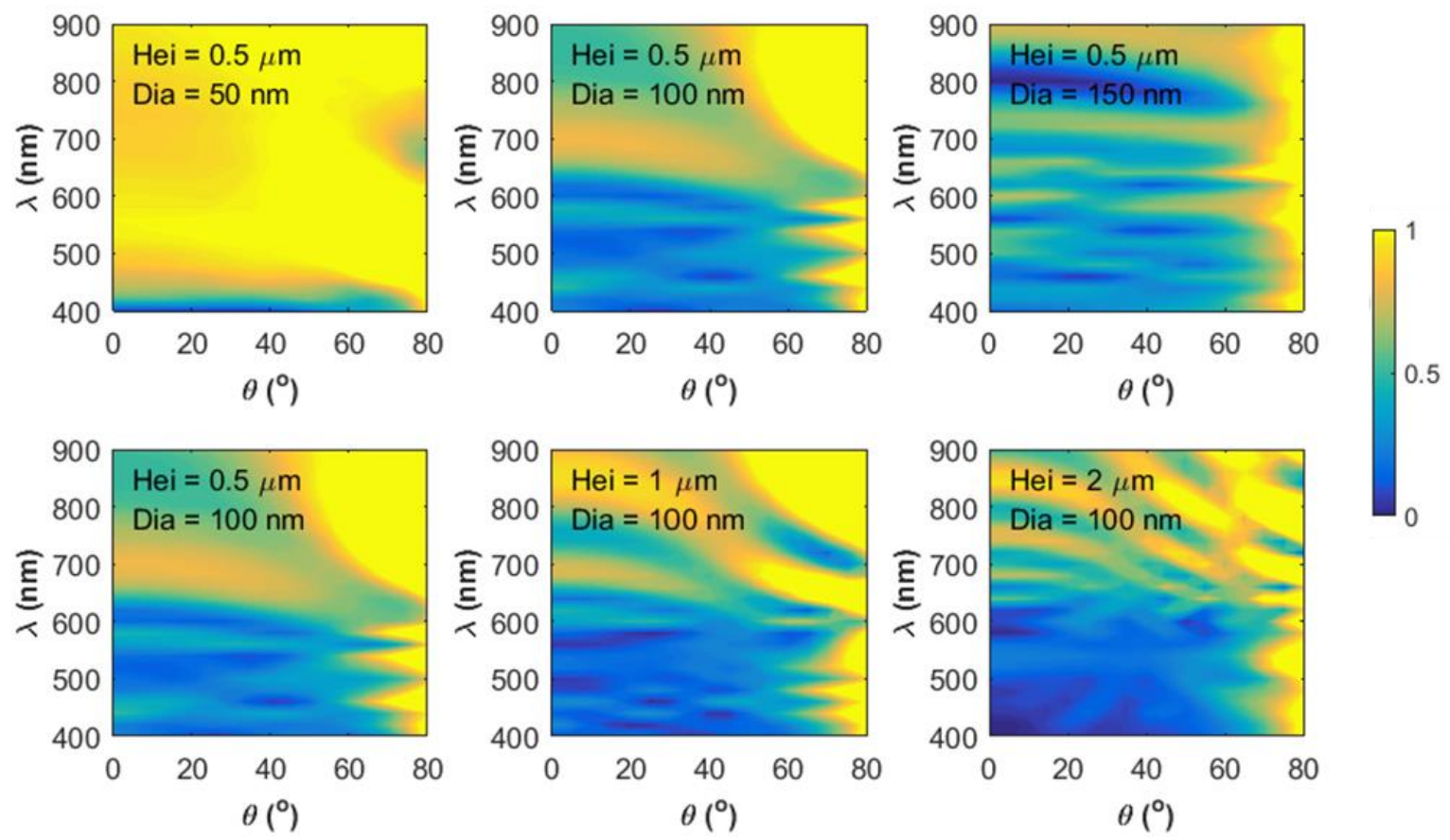

Figure 2:Reflection spectra (normalised to flat-Si) of a periodic NWA under varying angles, $\theta$, for a given height (Hei) and diameter (Dia)
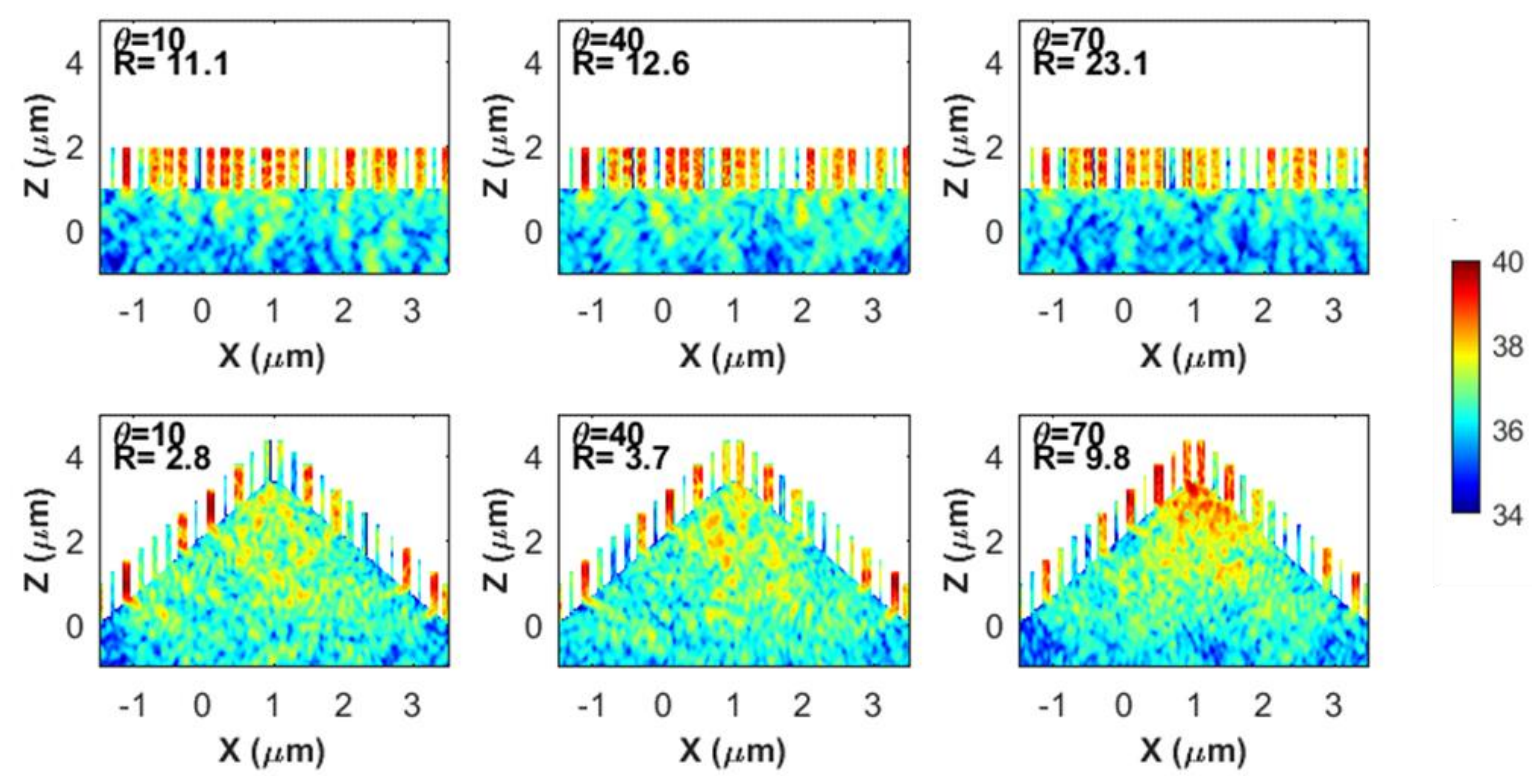

Figure 3: Spatially mapped power absorption (log-scale with A.U.) in cross-section through a pseudorandom NWA and hybrid structure for $\lambda=650 \mathrm{~nm}$ for varying $\theta$. Reflectivity (\%) is also stated for each case. 


\subsection{Experimental reflectivity}

Figure 4a. shows the reflectivity of a flat Si surface, random pyramids, silicon nanowires and the hybrid structure under normal incidence. The low reflectivity nature of the hybrid surface is observable with an average reflectance of less than $2 \%$. The average (mean for all wavelengths) total hemispherical reflectivity, normalised to polished silicon, of pyramid, nanowire and hybrid textured surface under varying angles of incidence from a broadband light source is presented in Figure 4b. The reflectivity is an average of results for $\mathrm{S}$ and $\mathrm{P}$ polarised input light.

The results show that across the angular range (whereby $8^{\circ}$ is the minimum angle of incidence), the NWA and hybrid textures outperform (i.e. exhibit lower reflectivity than) the pyramidal texture. The use of pyramids is a common light trapping technique as it can increase the optical path length of the incident light to several times the thickness of a substrate. The optical path length is defined as the distance travelled by light through a device and an increase in this with respect to the physical thickness of the device leads to more light absorption and so an increase in efficiency (provided the extra carriers generated can be collected). The incident light is trapped by the substrate as the angled (rough) surface will couple light obliquely into the semiconductor material and as such increases the optical path. When the geometry of the device approaches the wavelength of the incident spectrum, as in the case for the NWA and hybrid surface, the ray optic limit can be surpassed and additional light trapping mechanisms can be utilised to achieve further enhancements in the amount of light captured. The trends exhibited by the experimental results agree with the simulations, showing that the hybrid outperforms the NWA sample and retains low reflectivity for increasing $\theta$. The simulations underestimate the performance of these structures as the MACE NWs are much more heterogeneous than can be modelled using the pseudo-random method employed for the simulation in this study. Figure 5 shows the total hemispherical (normalised to planar Si) reflectivity across the wavelength range for the different surfaces under varying incident angles. The trend observed in the simulation for the NWA case, in which reflectivity for larger $\lambda$ increases more than shorter $\lambda$, with increasing $\theta$ is also evident in the experimental results. However, this trend is not so prevalent in the hybrid case whereby reflectivity for larger $\lambda$ remains low with increasing $\theta$. This feature of the hybrid surface can be very beneficial for solar cells which are specifically dependent on the longer wavelengths, e.g. rear contact silicon solar cells used as the bottom sub-cell in a tandem device.

(a)

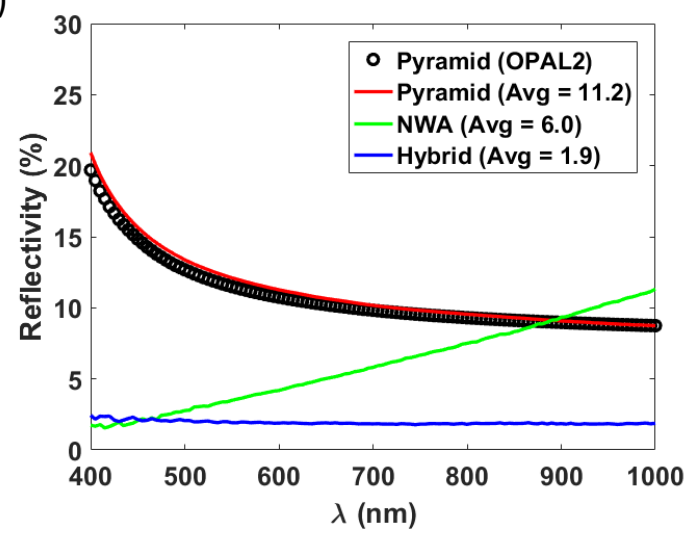

(b)

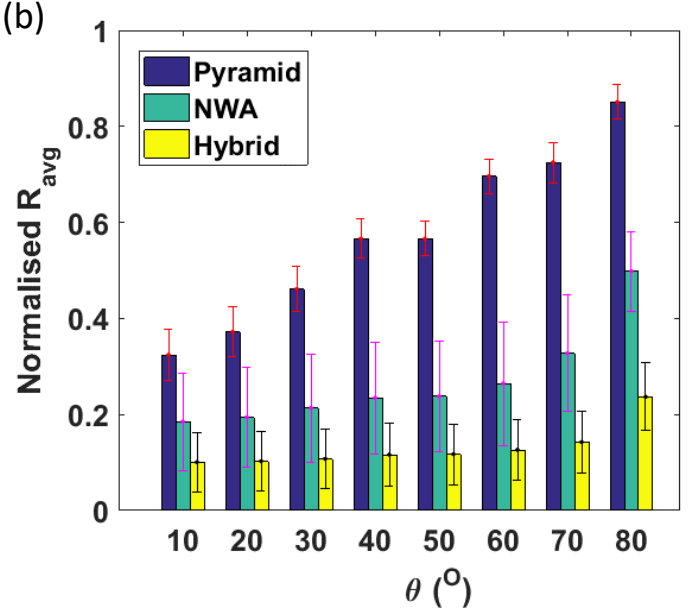

Figure 4: a) Reflectance spectra (400-1000 nm) of flat, pyramid, NWA and hybrid surfaces. Pyramid surface modelled on OPAL2 [34] is also shown. b) Average reflectivity (400 nm-900 nm), normalised to polished $\mathrm{Si}$, under varying incident angles for different surface textures. 

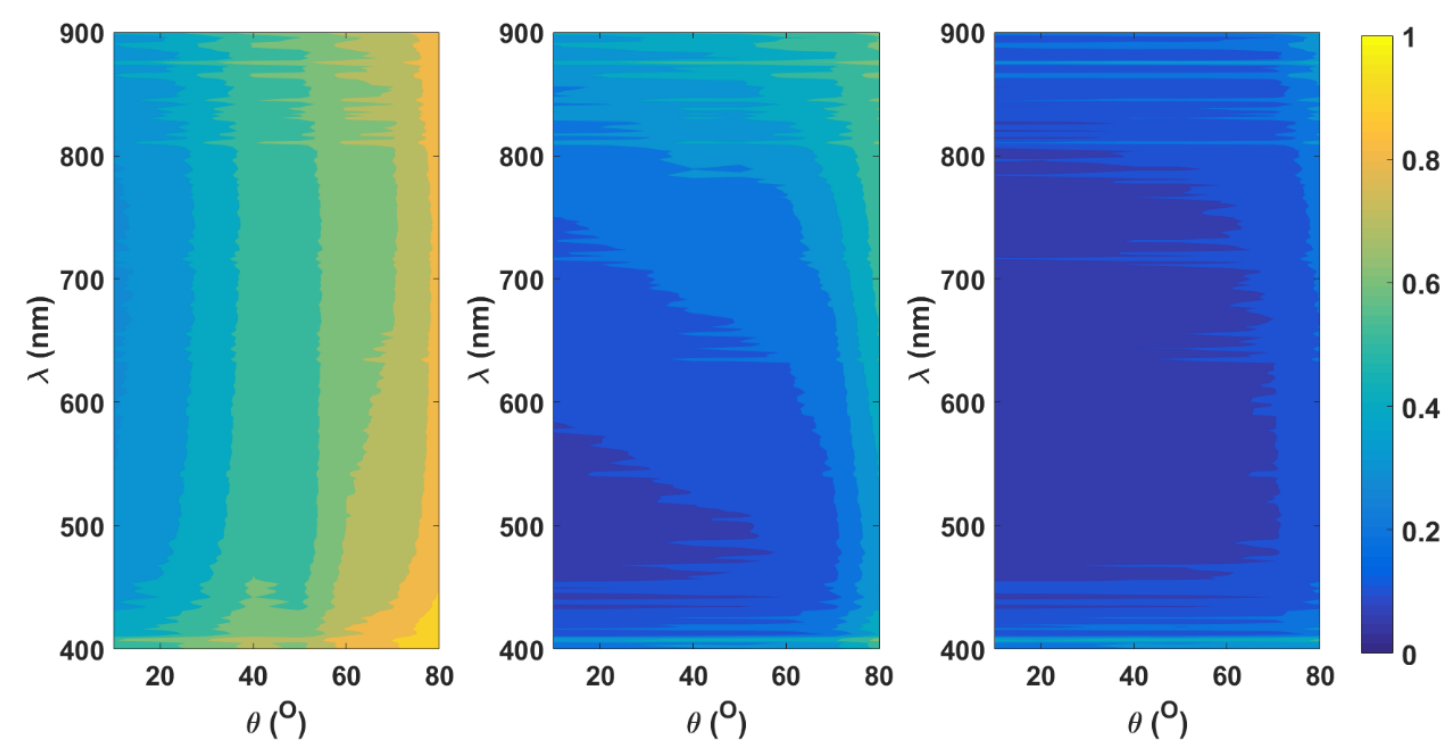

Figure 5: Normalised (to planar Si) reflectivity of pyramid (left), NWA (middle) and hybrid (right) surface under varying angles of incidence.

\section{Surface passivation}

Specimens for lifetime characterisation were fabricated using the pyramid, NWA and hybrid surface structuring, and compared to a reference flat specimen. All samples were coated with ALD alumina and characterised for morphology and recombination at the dielectric-silicon interface.

\subsection{Microscale morphology}

Figure 6 shows scanning electron microscopy (SEM) images of the fabricated silicon nanowires (NWs) (a), pyramids (b) and hybrid (c,d) surface with alumina coating. The nanowires vary in diameter from $30 \mathrm{~nm}$ to $200 \mathrm{~nm}$ and have heights of approximately $2 \mu \mathrm{m}$. The nanowires can be seen to bunch, an effect more prominent with the alumina coating. The bunching occurs as MACE is a water-based etching process, so during the drying step the NWs agglomerate in order to reduce surface tension, and are held together with Van der Waals forces [35]. Conformal coverage of the NWs with alumina using ALD was achieved by inserting a precursor diffusion time of $10 \mathrm{~s}$ for every $\AA$ of deposition, in order to achieve good exposure of the high surface area structures. The $\mathrm{Al}_{2} \mathrm{O}_{3}$ coating thickness of the structures is $15 \mathrm{~nm}$. The random pyramids, due to an etch time of $60 \mathrm{mins}$, are of high density (average of 131 pyramids within $120 \mu \mathrm{m}^{2}$ area) with base widths varying from sub-micron up to $5 \mu \mathrm{m}$. The hybrid surface shows that the NWs etch into the pyramidal facets are shorter than that of NWA on flat Si. This agrees with the literature and the reasons for shorter NWA are discussed in [13]. 


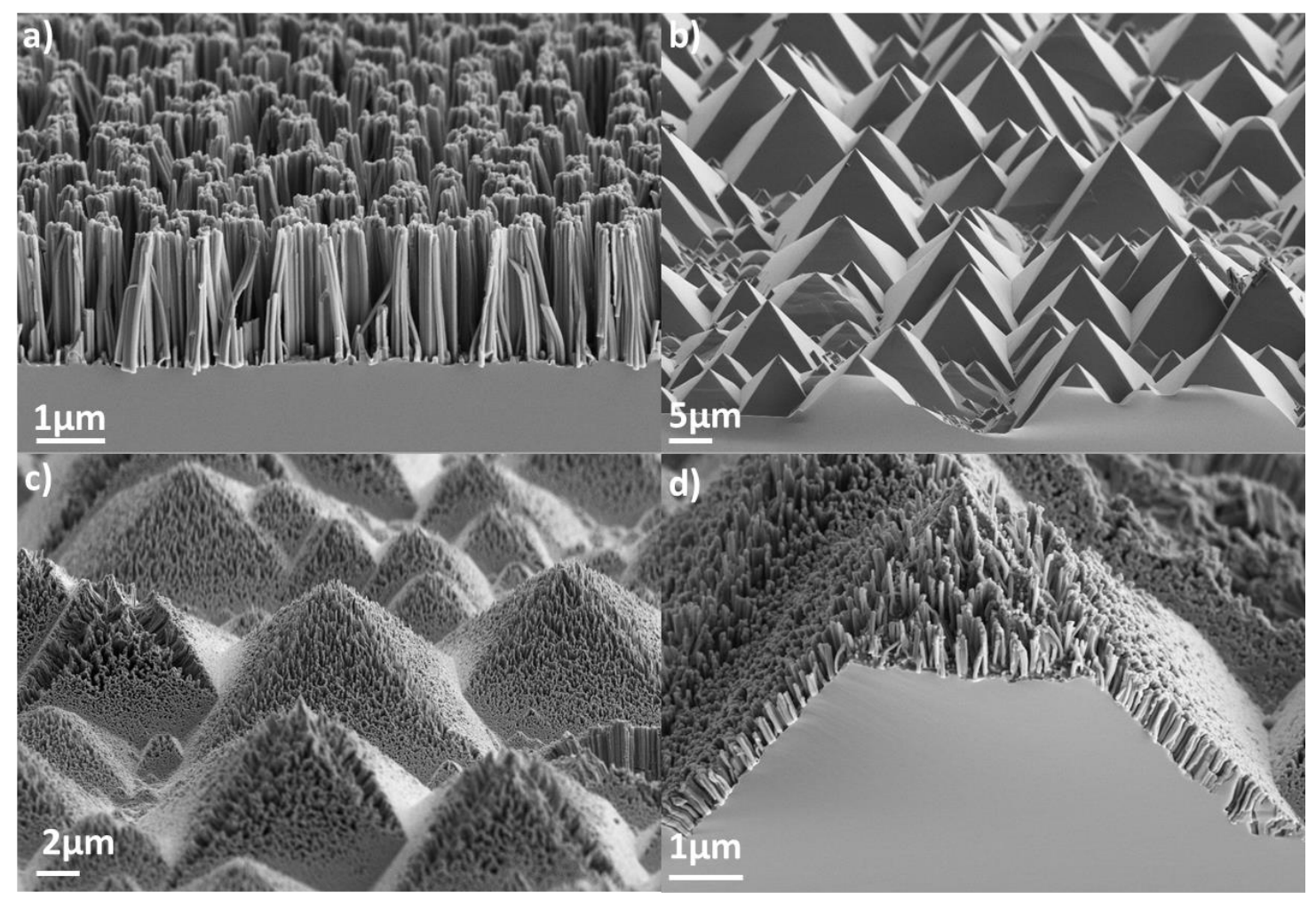

Figure 6: SEM images of silicon nanowire array (a), pyramidal (b) and hybrid (c) surface coated with alumina. Close up of hybrid surface shown in (d).

\subsection{Interface recombination}

The effective lifetime in alumina passivated samples was measured using photo-conductance decay. Surface passivation using ALD alumina was firstly studied on samples that did not undergo a post deposition anneal. Figure 7a illustrates the injection dependent minority carrier lifetime of these specimens before and after field effect passivation is enhanced extrinsically using corona discharge. These results demonstrate that, in flat and pyramidal surfaces, recombination can be effectively reduced by adding negative charge to alumina, and $\tau_{e f f}=5.6 \mathrm{~ms}$ and $0.4 \mathrm{~ms} @ \Delta \mathrm{n}=10^{15} \mathrm{~cm}^{-3}$ are possible in flat and pyramidal surfaces, respectively. The morphology study in the previous section showed that optimal conformity can be achieved on NWA structures when the optimized ALD alumina recipe is used. Despite this, lifetime measurements on NWA samples with the conformal coating without post deposition anneal, Figure 7b, show a very small degree of surface passivation for both NWA and hybrid surfaces -effective lifetimes of 40 and $25 \mu$ s respectively. When field effect passivation is enhanced by the use of negative corona discharge the effective lifetime only increases to 50 and $30 \mu s$ respectively. The use of corona discharge is only marginally advantageous in this case. 

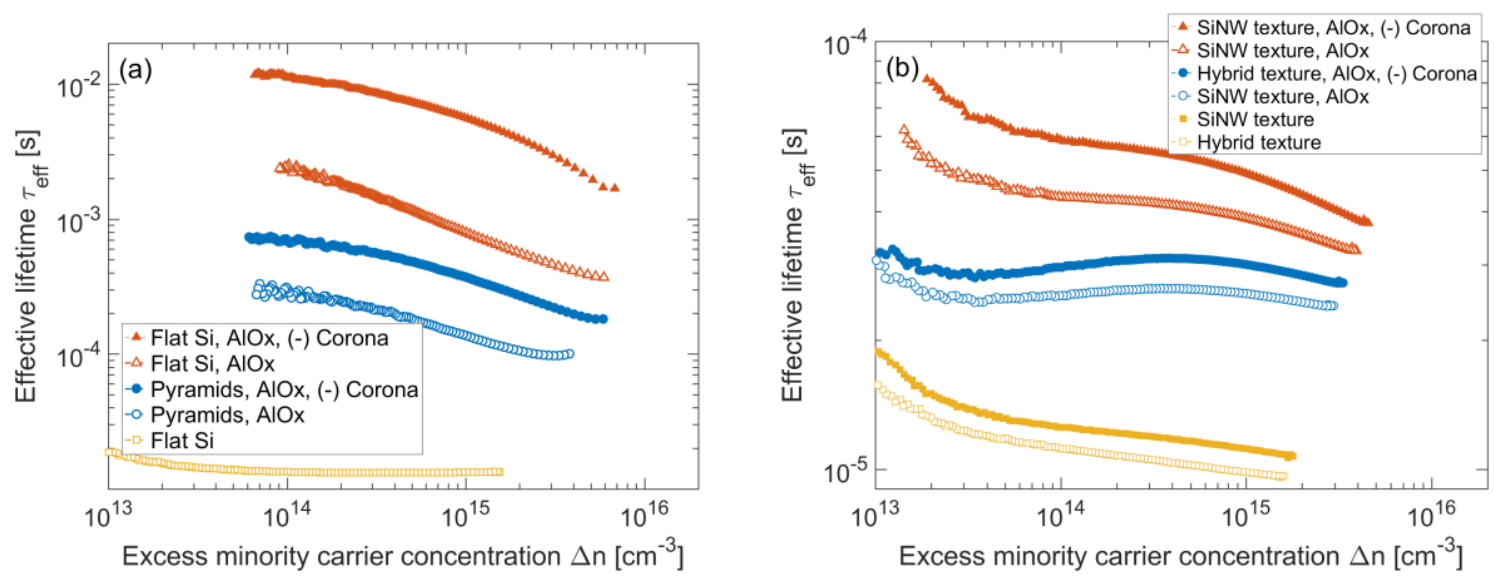

Figure 7. (a) Photo-conductance lifetime measurements of flat and pyramid textured Si bare surfaces, after alumina passivation deposition, and after optimal negative corona discharge, (b) Photoconductance lifetime measurements of NWA and hybrid NWA and Pyramid textured Si using an ALD alumina passivation coating as deposited and field effect passivated using corona charge.

A second study was done on a set of specimens processed equally to produce a flat, pyramid, NWA and hybrid structures coated with $\mathrm{Al}_{2} \mathrm{O}_{3}$ which were annealed after ALD deposition. This anneal resulted in a substantial improvement of the passivation quality. Figure 8 (a) illustrates the lifetime measurements in specimens fabricated with all four surface structures. An outstanding increase in lifetime was achieved in specimens bearing silicon nanowire structures. When the NWAs were used alone, effective lifetime increased to $1.21 \mathrm{~ms}$. In a hybrid surface it increased to $1.05 \mathrm{~ms}$. For flat and pyramidal surfaces, the increase in lifetime was similar to that achieved using extrinsic corona charge alone. Thus it is concluded that a large part of the improvement in passivation occurring after thermal treatment of ALD deposited alumina originates from increasing the concentration of negative charge in the films. This is in agreement with previous $\mathrm{ALD} \mathrm{Al}_{2} \mathrm{O}_{3}$ work performed in flat silicon [36]. For pyramidal surfaces, however, lower passivation quality is observed with respect to hybrid and SiNW surfaces. This could originate from the difference in chemical processing prior $\mathrm{ALD} \mathrm{Al}_{2} \mathrm{O}_{3}$ deposition, yet the reason was not investigated in detail since this work is mainly concerned with nanostructured surfaces. Negative corona charge had a marginal effect on the lifetime of all specimens shown in Figure 8 (a), and thus are not reported here. High frequency $\mathrm{CV}$ measurements were conducted on the flat $\mathrm{Al}_{2} \mathrm{O}_{3}$ coated specimens after the post deposition anneal, before and after corona discharge treatment. Corona treatment did not change the measured capacitance or flat band voltage, thus indicating that all corona deposited charge resided at the top surface of the dielectric. Figure $8 \mathrm{~b}$ illustrates a typical CV measurement including extraction of flat band voltage and interface state density as per the Terman method [37]. An ideal MOS capacitance curve has been included for comparison (solid line). Here it is observed that a 'stretch-out' in the voltage axis occurs for the experimental CV curve with respect to the theoretical curve. This indicates that the interface is poorly chemically passivated, yet the positive shift in flat-band voltage shows that a large concentration of insulator charge is providing outstanding field effect passivation. Additionally, the film is extremely leaky as observed by the dropdown in the accumulation regime.

To explain the lack of effectiveness of external charge in black silicon specimens it is proposed that, when using corona on nanowire structured surfaces, the charge is only deposited on a small area at the top of the high aspect ratio structures. This is illustrated schematically in Figure 8c. When a post deposition anneal is applied, the charge is generated very close to the insulator-semiconductor interface throughout the surface area. This effectively provides field effect passivation to the whole interface. If 
extrinsic methods of controlling charge in dielectrics are to be used -e.g. work in reference [38], the charged species must be present during film formation and only charged externally using a combination of temperature and electric field. This could be desirable if the polarity of charge were inverted such that no inversion region is created, for instance, at the top surface of an n-type back contacted cell.
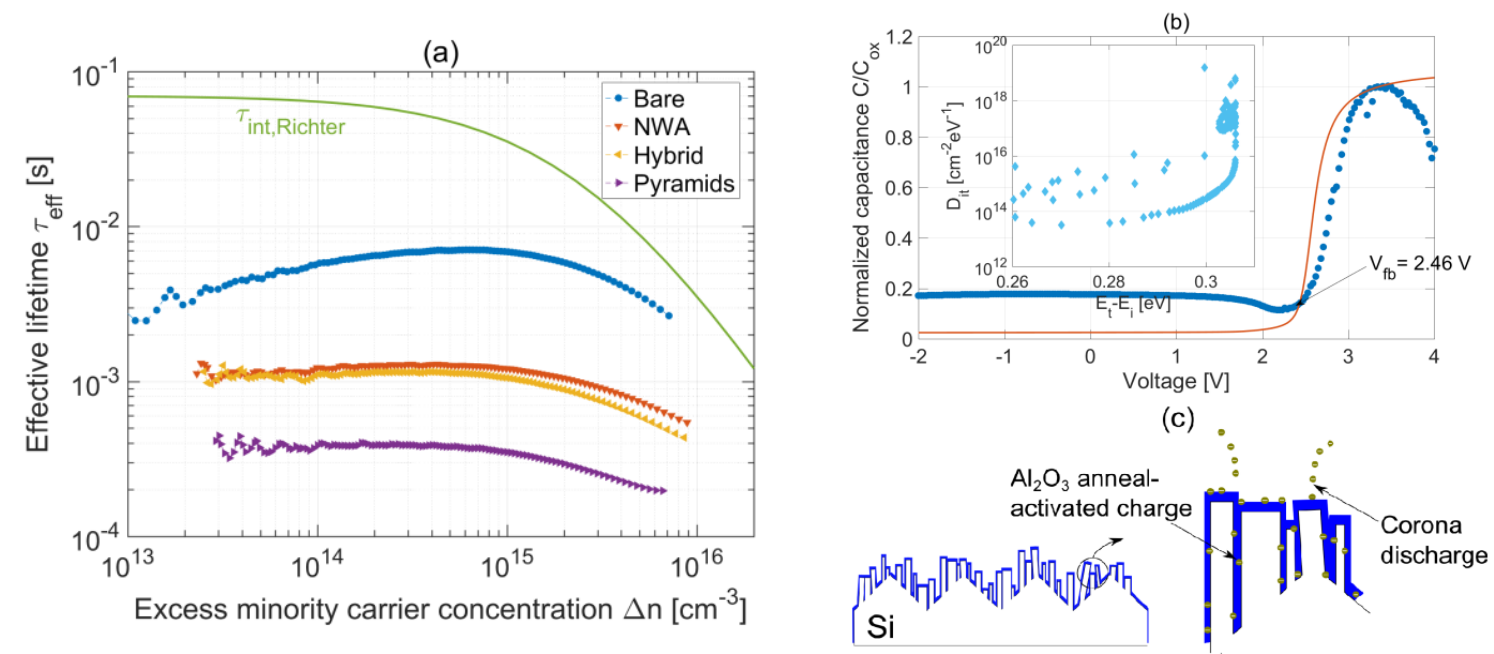

Figure 8. (a) Photo-conductance lifetime measurements of flat, pyramid, NWA and hybrid NWA and Pyramid textured Si using an ALD alumina passivation coating having a post deposition anneal to activate the passivation quality. (b) CV measurement including extraction of flat band voltage and interface state density. (c) Schematic of charge deposited on a small area at the top of the high aspect ratio structures.

Upper limits to surface recombination velocities were calculated as $S R V=W / 2\left(1 / \tau_{\text {eff }}-1 / \tau_{i}\right)$ using Richter's [39] parametrisation of intrinsic lifetime. Emitter saturation current densities were calculated using Kane and Swanson's [40] method as implemented in Sinton lifetime software ver 4.6.0. Table 1 illustrates these recombination parameters alongside the 1 sun implied $V_{o c}$ extracted from the Sinton software and the insulator charge $Q_{i n s}$, extracted from CV measurements for the annealed sample, and KP measurements for the corona charged samples. To the best of our knowledge, these are among the lowest observed recombination values for ultra-black silicon (low reflectivity across varying $\theta$ ) using activated alumina. These results exceed in passivation those achieved by Otto et al [21] and compare well to those shown by Savin et al [10], despite the difference in substrate type, dopant concentration and thickness. It is also evident that corona charge concentration on the top of the films is not as high in density as that estimated using CV after post deposition anneal. This can be due to the high leakage current measured using CV. It is also noted that outstanding passivation of flat silicon surfaces has been previously achieved by Richter [39], Werner [41] and Hoex [25], with SRV $<1.5 \mathrm{~cm} / \mathrm{s}$, yet the ALD alumina in this work has been specially optimised to provide good conformity of the high aspect ratio nanostructures. An activation anneal, however, has proven advantageous for effective passivation.

\begin{tabular}{|c|c|c|c|c|c|}
\hline Surface structure & $\begin{array}{c}\tau_{\text {eff }} \\
{[\mathrm{ms}]}\end{array}$ & $\begin{array}{l}S R V_{U L} \\
{[\mathrm{~cm} / \mathrm{s}]}\end{array}$ & $\begin{array}{c}J_{0 s} \\
{\left[\mathbf{f A} / \mathbf{c m}^{2}\right]}\end{array}$ & $\begin{array}{c}i V_{o c} \\
1-\operatorname{sun}[\mathrm{V}] \\
\end{array}$ & $\begin{array}{r}Q_{\text {ins }} \\
{\left[\mathbf{q} / \mathbf{c m}^{2}\right]} \\
\end{array}$ \\
\hline Flat/ $/ \mathrm{Al}_{2} \mathrm{O}_{3}+\mathrm{Neg}$ Corona & 5.64 & 4.84 & 54.2 & 0.682 & $-5.1 \times 10^{12}$ \\
\hline Flat $/ \mathrm{Al}_{2} \mathrm{O}_{3}+$ Anneal & 6.85 & 3.82 & 10.2 & 0.704 & $-8.1 \times 10^{12}$ \\
\hline Pyramid $/ \mathrm{Al}_{2} \mathrm{O}_{3}+$ Neg Corona & 0.37 & 86.4 & 466 & 0.614 & $-2.3 \times 10^{12}$ \\
\hline Pyramid $/ \mathrm{Al}_{2} \mathrm{O}_{3}+$ Anneal & 0.35 & 91.0 & 447 & 0.612 & - \\
\hline $\mathrm{NWA} / \mathrm{Al}_{2} \mathrm{O}_{3}+$ Anneal & 1.21 & 26.0 & 84.6 & 0.660 & - \\
\hline Hybrid/ $/ \mathrm{Al}_{2} \mathrm{O}_{3}+$ Anneal & 1.05 & 30.0 & 134 & 0.650 & - \\
\hline
\end{tabular}


Table 1. Summary of surface recombination parameters obtained from lifetime $\left(\Delta \mathrm{n}=10^{15} \mathrm{~cm}^{-3}\right), \mathrm{KP}$ and CV measurements.

\section{The effect of ultra-black silicon surfaces in IBC cells}

In order to quantify the potential efficiency improvement of the passivated hybrid surface over that of NWA and pyramidal surfaces, a device simulator (Quokka [42]) is utilised for modelling IBC silicon solar cells. This programme allows the design of an IBC cell and input of carrier generation profiles based on reflection measurements, as well as front surface $\mathrm{J}_{0}$ values based on lifetime measurements. The remaining input parameters of the IBC device are summarised in table 2 and the device models used follow those described in [43]. The simulated current-voltage results are shown in Figure 9. It can be seen that as $\theta$ increases, the change in $\mathrm{J}_{\mathrm{sc}}$ for the hybrid case is lower than that of NWA and pyramidal surfaces. This translates to an efficiency change from low $\theta$ to high $\theta$ of $14 \%, 5 \%$ and $1 \%$ for pyramidal, NWA and hybrid surfaces respectively. The device modelling illustrates that the ability to retain low reflectivity at high $\theta$ can indeed prevent a significant reduction in efficiency. The low $\mathrm{V}_{\text {oc }}$ observed, particularly for the pyramidal surface, shows that further improvement of the passivation is needed for the front surface $\mathrm{J}_{0}$. To explore the potential performance of the hybrid surface on an IBC cell further, Figure 10a illustrates the efficiency as a function of front surface $\mathrm{J}_{0}$. The results show that reducing front surface $\mathrm{J}_{0}$ down to $10 \mathrm{fA} \mathrm{cm}^{-2}$ can lead to efficiencies exceeding $23 \%$. To relate this against current technology used to reduce reflections on solar cells, the results are compared against a pyramidal surface with an optimised antireflective coating (ARC) stack of $80 \mathrm{~nm} \mathrm{SiO}$ and $70 \mathrm{~nm} \mathrm{SiN}$ (carrier generation profile taken from OPAL2). A front surface $\mathrm{J}_{0}$ of $10 \mathrm{fA} \mathrm{cm}^{-2}$ is used in the Quokka model and the results for efficiency against varying incident angle are shown in Figure 10b. The chart shows that at low $\theta$ both surfaces produce comparable efficiency, however as $\theta$ increases a significant drop is observed in the pyramidal+ARC case whilst the hybrid remains at a high efficiency level. This illustrates the advantage that such hybrid surfaces can have over current light trapping technology. This remains true for an encapsulated cell, although to a lesser extent as the incident angular range is limited by refraction at the glass/air interface. Furthermore, the hybrid surface discussed here does not contain an optimised ARC stack, an addition that could lead to further improvements in efficiency. 


\begin{tabular}{|l|l|l|l|l|l|l|}
\hline \hline \multicolumn{3}{|l|}{ Geometry } & \multicolumn{2}{l|}{ Conductive Boundary } & \multicolumn{2}{l|}{ Bulk Properties } \\
\hline $\begin{array}{l}\text { Cell } \\
\text { thickness }\end{array}$ & $200 \mu \mathrm{m}$ & $\begin{array}{l}\text { Emitter } \\
\text { resistance }\end{array}$ & sheet & $160 \Omega / \square$ & Resistivity & $1.5 \Omega$ \\
\hline $\begin{array}{l}\text { Emitter } \\
\text { width }\end{array}$ & $320 \mu \mathrm{m}$ & Base sheet resistance & $30 \Omega / \square$ & Lifetime & $7 \mathrm{~ms}$ \\
\hline Base radius & $15 \mu \mathrm{m}$ & Emitter $\mathrm{J}_{0}-$ contacted & $\begin{array}{l}1200 \\
\mathrm{fA} / \mathrm{cm}^{2}\end{array}$ & Auger & Richter2012 \\
\hline $\begin{array}{l}\text { Contact } \\
\text { radius }\end{array}$ & $5 \mu \mathrm{m}$ & Emitter $\mathrm{J}_{0}-$ passivated & $30 \mathrm{fA} / \mathrm{cm}^{2}$ & Mobility & Klassen & \\
\hline $\begin{array}{l}\text { Contact } \\
\text { Pitch }\end{array}$ & $70 \mu \mathrm{m}$ & Base $\mathrm{J}_{0}-$ contacted & $\begin{array}{l}200 \\
\mathrm{fA} / \mathrm{cm}^{2}\end{array}$ & $\mathrm{~N}_{\mathrm{i}, \text { eff }}$ & $\begin{array}{l}9.65 \times 10^{9} \\
\mathrm{~cm}^{-3}\end{array}$ & \\
\hline & & Base $\mathrm{J}_{0}-$ passivated & $\begin{array}{l}170 \\
\mathrm{fA} / \mathrm{cm}^{2}\end{array}$ & & \\
\hline \hline
\end{tabular}

Table 2. Summary of the input parameters for the IBC model using the device simulator Quokka. The front surface $\mathrm{J}_{0}$ is based on experimental results shown in Table 1 . The unit cell model is shown in the last column. $\mathrm{P}$ and $\mathrm{N}$ regions shown in blue and red respectively, whilst green is a non-conductive boundary.
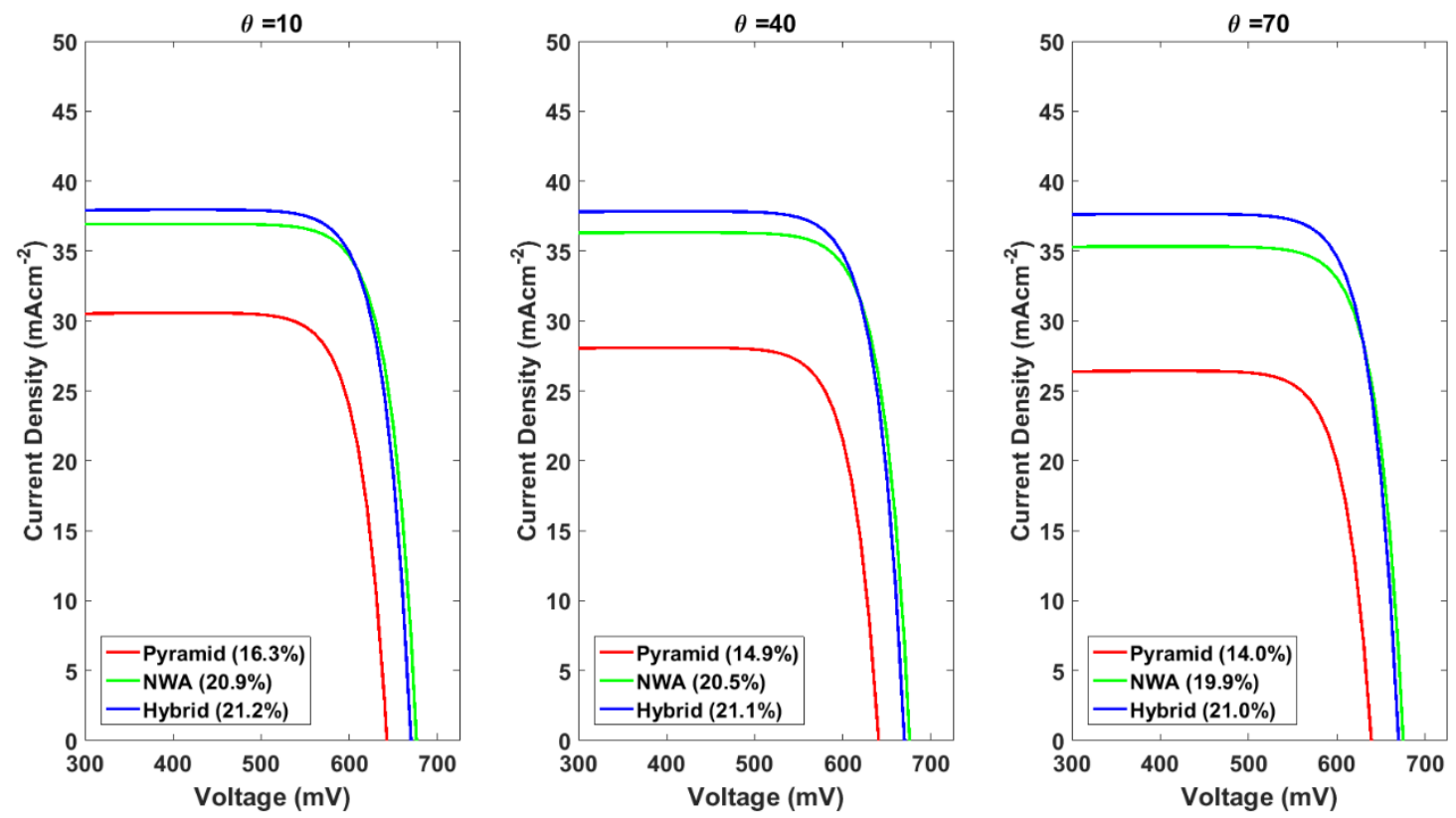

Figure 9: The current-voltage curve solved using Quokka comparing pyramid surface, NWA surface and hybrid surface on an IBC cell for varying incident angle of light 
a)

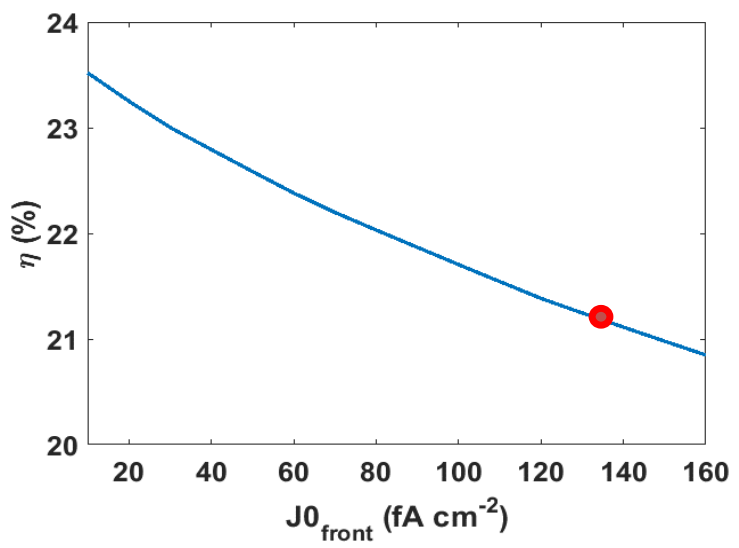

b)

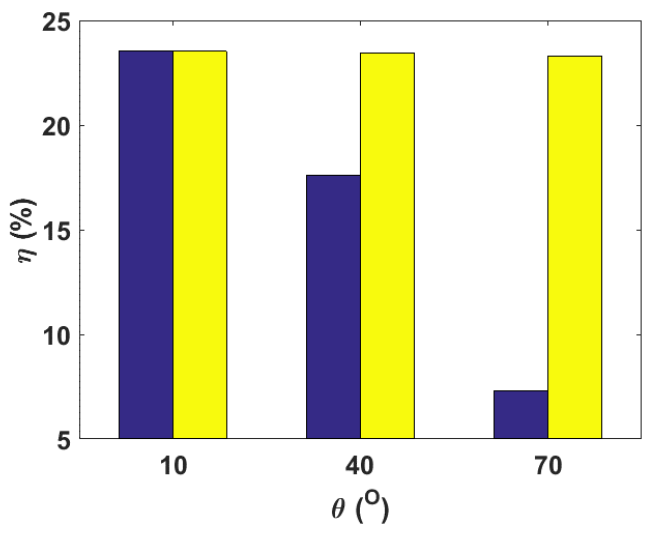

Figure 10: a) Efficiency improvement of IBC cell with front hybrid surface with reducing front surface $\mathrm{J}_{0}$. The red spot corresponds to front surface $\mathrm{J}_{0}$ of the hybrid surface passivated with alumina as shown experimentally in this work. b) Efficiency values, modelled using Quokka, for IBC cells with Pyramidal surface with ARC layer (blue) and hybrid surface (yellow) for varying incident angles

\section{Conclusion}

Ultra-low reflectivity $(<2 \%)$ was achieved by texturing a silicon surface with a hybrid structure of pyramids decorated with dense, vertically-aligned nanowire arrays, using cheap, scalable wet-etching processes. Studying the total hemispherical reflectivity under varying incident angles has revealed that the hybrid surface retains low reflectivity at larger $\theta$ compared to pyramid and NWA surfaces. FDTD modelling also supports these results with the superior performance of the hybrid surface attributed to the combination of the optical benefits of the pyramid (multiple reflections) and the NWA (guided modes and Fabry-Perot resonances). To passivate the textured surfaces, a conformal coating of alumina was formed on the hybrid structure by increasing the precursor diffusion time in the ALD process to 10s per $\AA$ of deposition. Lifetime measurements have shown that effective passivation in planar and pyramidal textured $\mathrm{Si}$ is possible using non-annealed alumina and extrinsically enhanced field effect passivation. Passivation of the NWA and hybrid structures was achieved by applying a post alumina deposition anneal with effective lifetime increased to $1.21 \mathrm{~ms}$ for the NWA and $1.05 \mathrm{~ms}$ for the hybrid surface. Device simulations in Quokka, using carrier generation profiles based on reflection measurements and front surface $\mathbf{J}_{0}$ based on passivation measurements, have shown that incorporation of the hybrid surface onto an IBC cell can result in power conversion efficiencies exceeding $21 \%$. The hybrid surface shows good retention of efficiency across all angles of incidence, with only a $1 \%$ reduction between $\theta=10^{\circ}$ and $\theta=70^{\circ}$. Furthermore, the simulations indicate that reducing front surface $\mathrm{J}_{0}$ down to $10 \mathrm{fA} \mathrm{cm}^{-2}$ can result in efficiencies exceeding $23 \%$, which is retained at high incident angles and therefore significantly outperforms an optimised pyramid surface with ARC layers.

\section{Acknowledgements}

T. Rahman and S.A. Boden acknowledge support from the Supersolar Solar Energy Hub (EPSRC grants EP/J017361/1 and EP/M014797/1). RS Bonilla is the recipient of an EPSRC (UK) Postdoctoral Research Fellowship, EP/M022196/1. PR Wilshaw acknowledges the support from EPSRC grant EP/M024911/1. All data supporting this study are openly available from the University of Southampton repository at http://dx.doi.org/10.5258/SOTON/397727. 


\section{References}

1. Shen, M., et al., High-density regular arrays of nanometer-scale rods formed on silicon surfaces via femtosecond laser irradiation in water. Nano letters, 2008. 8(7): p. 2087-2091.

2. Vorobyev, A. and C. Guo, Direct creation of black silicon using femtosecond laser pulses. Applied Surface Science, 2011. 257(16): p. 7291-7294.

3. Hsu, C.-M., et al., Wafer-scale silicon nanopillars and nanocones by Langmuir-Blodgett assembly and etching. Applied Physics Letters, 2008. 93(13): p. 133109.

4. Morton, K.J., et al., Wafer-scale patterning of sub-40 $\mathrm{nm}$ diameter and high aspect ratio (> 50: 1) silicon pillar arrays by nanoimprint and etching. Nanotechnology, 2008. 19(34): p. 345301.

5. $\mathrm{Li}, \mathrm{X}$. and P. Bohn, Metal-assisted chemical etching in HF/H2O2 produces porous silicon. Applied Physics Letters, 2000. 77(16): p. 2572-2574.

6. Peng, K.Q. and S.T. Lee, Silicon nanowires for photovoltaic solar energy conversion. Advanced Materials, 2011. 23(2): p. 198-215.

7. Sivakov, V., et al., Realization of vertical and zigzag single crystalline silicon nanowire architectures. The Journal of Physical Chemistry C, 2010. 114(9): p. 3798-3803.

8. Zhang, M.-L., et al., Preparation of large-area uniform silicon nanowires arrays through metalassisted chemical etching. The Journal of Physical Chemistry C, 2008. 112(12): p. 4444-4450.

9. Zhong, X., et al., Unveiling the formation pathway of single crystalline porous silicon nanowires. ACS applied materials \& interfaces, 2011. 3(2): p. 261-270.

10. Savin, $\mathrm{H}_{\text {., }}$ et al., Black silicon solar cells with interdigitated back-contacts achieve $22.1 \%$ efficiency. Nature nanotechnology, 2015.

11. Sopori, B., et al., Surface Damage Introduced by Diamond Wire Sawing of Si Wafers: Measuring in-depth and the Lateral Distributions for Different Cutting Parameters. MRS Proceedings, 2015. 1770: p. 61-66.

12. Ingenito, A., O. Isabella, and M. Zeman, Nano-cones on micro-pyramids: modulated surface textures for maximal spectral response and high-efficiency solar cells. Progress in Photovoltaics: Research and Applications, 2015. 23(11): p. 1649-1659.

13. Rahman, T., M. Navarro-Cía, and K. Fobelets, High density micro-pyramids with silicon nanowire array for photovoltaic applications. Nanotechnology, 2014. 25(48): p. 485202.

14. Hochbaum, A.I., et al., Controlled growth of Si nanowire arrays for device integration. Nano letters, 2005. 5(3): p. 457-460.

15. Choi, W., et al., Synthesis of silicon nanowires and nanofin arrays using interference lithography and catalytic etching. Nano letters, 2008. 8(11): p. 3799-3802.

16. Cloutier, S.G., et al., Enhancement of Radiative Recombination in Silicon via Phonon Localization and Selection-Rule Breaking. Advanced Materials, 2006. 18(7): p. 841-844.

17. Oh, J., H.-C. Yuan, and H.M. Branz, An 18.2\%-efficient black-silicon solar cell achieved through control of carrier recombination in nanostructures. Nat Nano, 2012. 7(11): p. 743-748.

18. Fang, X., et al., Ultrathin interdigitated back-contacted silicon solar cell with light-trapping structures of Si nanowire arrays. Solar Energy, 2015. 116: p. 100-107.

19. Mack, S., et al., Silicon surface passivation by thin thermal oxide/PECVD layer stack systems. Photovoltaics, IEEE Journal of, 2011. 1(2): p. 135-145.

20. Davidsen, R.S., et al., Black silicon laser-doped selective emitter solar cell with $18.1 \%$ efficiency. Solar Energy Materials and Solar Cells, 2016. 144: p. 740-747.

21. Otto, M., et al., Extremely low surface recombination velocities in black silicon passivated by atomic layer deposition. Applied Physics Letters, 2012. 100(19): p. 191603.

22. Otto, M., et al., Passivation of Optically Black Silicon by Atomic Layer Deposited Al 20 3. Energy Procedia, 2013. 38: p. 862-865.

23. Davidsen, R.S., et al., Angle resolved characterization of nanostructured and conventionally textured silicon solar cells. Solar Energy Materials and Solar Cells, 2015. 140: p. 134-140.

24. Monsma, D. and J. Becker, The Savannah ALD System-An Excellent Tool for Atomic Layer Deposition. Material Matters, 2006. 1(5). 
25. Hoex, B., et al., On the c-Si surface passivation mechanism by the negative-charge-dielectric Al2O3. Journal of Applied Physics, 2008. 104(11): p. 113703.

26. Bonilla, R.S., F. Woodcock, and P.R. Wilshaw, Very low surface recombination velocity in $n$ type c-Si using extrinsic field effect passivation. Journal of Applied Physics, 2014. 116(5): p. 054102.

27. Sinton, R.A., A. Cuevas, and M. Stuckings. Quasi-steady-state photoconductance, a new method for solar cell material and device characterization. in Photovoltaic Specialists Conference, 1996., Conference Record of the Twenty Fifth IEEE. 1996. IEEE.

28. Bonilla, R.S., et al., Stable, extrinsic, field effect passivation for back contact silicon solar cells. Solid State Phenomena, 2016. 242.

29. Deinega, A. and S. John, Effective optical response of silicon to sunlight in the finite-difference time-domain method. Optics letters, 2012. 37(1): p. 112-114.

30. Sturmberg, B.C., et al., Modal analysis of enhanced absorption in silicon nanowire arrays. Optics Express, 2011. 19(105): p. A1067-A1081.

31. Alaeian, H., A.C. Atre, and J.A. Dionne, Optimized light absorption in Si wire array solar cells. Journal of Optics, 2012. 14(2): p. 024006.

32. Lumerical, F., Solutions. Web source [https://www. lumerical. com/tcad-products/fdtd/], 2012.

33. Balanis, C.A., Advanced Engineering Electromagnetics. Wiley, 2012.

34. Baker-Finch, S.C. and K.R. McIntosh. A freeware program for precise optical analysis of the front surface of a solar cell. in Photovoltaic Specialists Conference (PVSC), 2010 35th IEEE. 2010. IEEE.

35. Schmidt, M.S., J. Hübner, and A. Boisen, Large Area Fabrication of Leaning Silicon Nanopillars for Surface Enhanced Raman Spectroscopy. Advanced Materials, 2012. 24(10): p. OP11-OP18.

36. Dingemans, G., et al., Influence of the oxidant on the chemical and field-effect passivation of Si by ALD A/2O3. Electrochemical and Solid-State Letters, 2011. 14(1): p. H1-H4.

37. Terman, L.M., An investigation of surface states at a silicon/silicon oxide interface employing metal-oxide-silicon diodes. Solid-State Electronics, 1962. 5(5): p. 285-299.

38. Bonilla, R.S. and P.R. Wilshaw, A technique for field effect surface passivation for silicon solar cells. Applied Physics Letters, 2014. 104(23): p. 232903.

39. Richter, A., et al., Improved quantitative description of Auger recombination in crystalline silicon. Physical Review B, 2012. 86(16): p. 165202.

40. Kane, D. and R. Swanson. Measurement of the emitter saturation current by a contactless photoconductivity decay method. in IEEE photovoltaic specialists conference. 18. 1985.

41. Werner, F., et al., Very low surface recombination velocities on $p$-and n-type c-Si by ultrafast spatial atomic layer deposition of aluminum oxide. Applied Physics Letters, 2010. 97(16): p. 162103.

42. Fell, A., $A$ free and fast 3D/2D solar cell simulator featuring conductive boundary and quasineutrality approximations. IEEE Transactions on Electron Devices, 2012. 60(2): p. 733-738.

43. Fell, A., et al., Input parameters for the simulation of silicon solar cells in 2014. Photovoltaics, IEEE Journal of, 2015. 5(4): p. 1250-1263. 